erkennbare Reaktionart eines betroffenen Individuums; während sie bei Infektionen mit Erregern höherer biologischer Ordnungsklassen eine unter anderen möglichen Ursachen für eine ,,stille Feiung“" darstellt, ist sie hier deren obligate Voraussetzung.

\title{
II. Beispiele für das Vorliegen inapparenter Virus- und Rickettsieninfektionen
}

Die ersten Bemühungen um das Problem der inapparenten Infektion gehen, wie bereits gezeigt wurde, um Jahrzehnte zurück. Die Nachweise und die genauere Verfolgung solcher Prozesse häufen sich aber erst in den letzten Jahren. Das hängt vordringlich mit der Entwicklung der experimentellen Laboratoriumstechnik zusammen; erst durch den Austausch des kostspieligen Tierversuchs mit den verschiedenen Ei- und Gewebekulturtechniken sind die wirtschaftlichen Voraussetzungen geschaffen worden, um auf breiterer Basis Virusisolierungen und teilweise auch Antikörpernachweise durchzuführen.

Wenn wir nun Beispiele für inapparente Virus- und Rickettsieninfektionen aufführen wollen, so interessieren uns in allererster Linie jene Wirt-Erreger-Beziehungen, bei welchen ein symptomloser oder abortiver Reaktionsablauf häufiger vorkommt. Die Infektionen mit einem hohen Kontagionsindex [244], bei denen also die Infektion ziemlich gleichbedeutend mit einer Erkrankung ist, und wie sie bei einem Teil der sogenannten exanthematischen Viruskrankheiten (Pocken, Masern, Varicellen) vorliegen, sind in diesem Zusammenhang von geringerer Bedeutung und können im Rahmen dieser Abhandlung aus naheliegenden Gründen kursorisch behandelt werden. Am Beispiel der Masern soll dies begründet und gerechtfertigt werden.

\section{Die Frage der inapparenten Maserninfektion als Beispiel für eine Virusinfektion mit hohem Kontagionsindex}

Für diese Infektion ist in den Untersuchungen von PaNum [519] und v. HoFF [301] gelegentlich zweier Inselepidemien auf den Färöer Inseln in den Jahren 1846 und 1875, sowie von Pfeilsticker [543] im Jahre 1863 in Hageloch bei Tübingen ein Kontagionsindex um 95\% ermittelt worden. Neuere gleichartige Untersuchungen in abgeschlossenen Arealen, in welche das Masernvirus eingeschleppt worden war, sind von CHRISTENSEN u. Mitarb. [130] sowie von Peart u. Nagler [530] mitgeteilt worden; dabei wurden in bis dahin masernfreien arktischen Gebieten Morbiditätsziffern von 99-99,9\% errechnet. Es ist nun aber keineswegs der Schluß berechtigt, daß bei den verbleibenden $0,1-5 \%$ vermutlich kontagiierter, aber nicht erkrankter Personen obligat eine inapparente Infektion 
abläuft. Vielmehr treten hier noch Ursachen hinzu, welche - von DE RUDDER [588, 589] ausführlich abgehandelt - in unseren nachfolgenden Betrachtungen der Gründe von Apparenz und Inapparenz einer Infektion zu besprechen sind; im Augenblick hat uns lediglich zu interessieren, ob überhaupt die Annahme inapparenter Maserninfektionen berechtigt ist. ,Morbili sine exanthemate“ können deshalb nicht so ohne weiteres einbezogen werden, weil zu ihrer Anerkennung der Nachweis Koplikscher Flecken und eines Enanthems gefordert werden $[199 ; 29 \%$; 723; u. a. m.]. Aufschlußreich im Sinne einer Bejahung inapparenter Maserninfektionen ist eine Studie von Stocks u. KarN [666]; diese Autoren teilen mit, daß Kinder aus Häusern, in denen ein Masernfall vorgekommen war, ohne selbst Masern gehabt zu haben, in zwei kontrollierten Jahresfristen weniger empfänglich für eine Masernerkrankung gewesen seien als Kontrollkinder aus masernfreien Häusern. Eine durch virologische und immunologische Untersuchungen gesicherte Prüfung der Frage spontaner inapparenter Maserninfektionen des Menschen steht aber noch aus; bei der hohen Morbiditätsrate dieser Erkrankung müßten solche Studien um signifikant zu sein - ein außerordentlich großes Kollektiv erfassen. Der grundsätzliche Beweis für die Möglichkeit einer unterschwellig bleibenden Infektion mit dem Masernvirus ist allerdings für den Affen erstmalig von ANDERson u. Goldberger [11] erbracht und von Nicolle u. CoNSEIL [502] insofern nachdrücklich bestätigt worden, als nach diesen Autoren das Blut infizierter, aber nicht masernerkrankter Tiere beim ungemaserten Menschen die Krankheit auslöste. Und seit der von NICoLLE u. CoNSEIL inaugurierten, aber erst von DEGKwITz [153, 154] eingeführten Prophylaxe mit Masernrekonvaleszentenserum, bzw. der daraus entwickelten Simultan-Immunisierung mit Masernvirus und Rekonvaleszentenserum im Sinne einer „Halbdrosselung “ oder ,Spätdrosselung“ [539], oder aber auch den Versuchen einer Masernverhütung durch Gamma-Globulinverabreichung [z. B. 126; 132] wird über die künstliche Erzeugung mitigierter Masern versucht, den Weg zu einer inapparenten Infektion $\mathrm{zu}$ finden. Hier wechselt die Problematik aber wiederum in Fragen nach den Ursachen einer Inapparenz hinüber; wir können sie vorerst unberührt lassen.

Wenden wir uns dagegen den Infektionen mit niedrigeren Kontagionsindices $\mathrm{zu}$, so häufen sich die interessierenden Beobachtungen und Befunde. Zuerst soll dabei von einer erwiesenen Infektion auf ihren Effekt geschlossen werden.

\section{Der Schluß von einer erwiesenen Infektion auf ihren Effekt}

Im Prinzip hat Jenner [333] im Jahre 1796 mit der Kuhpockenimpfung den prophylaktischen Effekt einer inapparenten Infektion vorweggenommen; es darf dabei allerdings nicht vergessen werden, daß 
korrekterweise weder das Kuhpockenvirus mit dem Pockenvirus identifiziert werden kann, noch daß die Kuhpockenvaccinierung - mit Eruption der Impfpusteln - eine inapparente Infektion sensu strictiori darstellt. Durch Sternberg [665] wurde dann aber 1892 und durch Béclère, Chambon u. MÉnard [52] 1896 und 1899 am Rind und Kalb die protektive Wirkung des Serum vaccine-immuner Tiere gegen eine nachträgliche Infektion bzw. die Virusneutralisation in vitro gezeigt.

Einen drastischen Modellbeweis für die inapparente Infektion haben Blanc, Caminopetros u. Manoussakis [76] im Jahre 1928 erbracht. Eine Überimpfung des Blutes $\left(3,5 \mathrm{~cm}^{3}\right)$ von einem Dengue-Kranken auf einen anderen Menschen blieb ohne klinischen Effekt. Es konnte aber bei diesem Probanden eine Virämie nachgewiesen werden; das am 3. Tag p. inf. von ihm entnommene Blut $\left(7 \mathrm{~cm}^{3}\right)$ erzeugte bei Übertragung auf eine dritte Versuchsperson das typische Dengue-Krankheitsbild. Die nachfolgende Ausbildung eines Immunitätsschutzes konnte bei dem inapparent Infizierten dadurch unter Beweis gestellt werden, daß bei einer Wiederholung des Versuches an ihm eine Virämie nicht mehr nachweisbar war. Die inapparente Dengue-Infektion ist auch von TANIGUCHI u. Mitarb. [676] beim Affen nachgewiesen worden. Bei diesen an 14 Cyclopusaffen durchgeführten Untersuchungen wurde der Beweis der Virämie dadurch erbracht, daß die Rückübertragung von Affenblut auf Menschen in 9 von 11 Versuchen eine Dengue-Erkrankung der Versuchspersonen zur Folge hatte; der interessante Befund, daß bei klinischer Inapparenz bei 3 der infizierten Affen anatomisch das Korrelat myokarditischer und glomerulitischer Veränderungen nachgewiesen werden konnte, wird später besprochen werden.

Grundsätzlich gleichartigen Charakter - in Analogie zu Nicolles Grundversuch mit Flecktyphusblut - hat das Experiment von PlowRIGHT [550], in welchem Schafe mit Rinderpestvirus infiziert worden waren. Die einzige Reaktion der infizierten Tiere bestand in einer kurzdauernden Temperaturerhöhung. Das Blut der Schafe war mit einem Maximum um den 5./6. Tag p. inocul. bis zum 9. Tag infektiös; der Virusnachweis aus dem Blut gelang nach dem 13. Tage nicht mehr.

Anderson [14] schloß auf Grund von Versuchen an über 40 Mädchen ohne Röteln-Anamnese, auf welche das Rötelnvirus durch Sprayung von Rachenspülflüssigkeit einer kranken Person übertragen worden war, und wobei nur etwa die Hälfte der Probanden erkrankte, die erworbene Immunität aber durchweg gut war, daß es wahrscheinlich subklinische, symptomlose Röteln gebe, welche ebenfalls Immunität hinterließen.

Auch mit dem Mumpsvirus ließ sich die Existenz einer inapparenten Infektion des Menschen beweisen [285]. Es wurde 15 Kindern amnionpassiertes Mumpsvirus inoculiert. Während bei 6 Kindern eine Parotitis 
und in einem Fall eine Orchitis auftrat, blieben bei 8 Kindern jegliche Krankheitszeichen aus, obwohl bei ihnen ebenso wie bei den Erkrankten nach der Infektion ein beträchtlicher Anstieg von Mumps-Antikörpern im Serum nachweisbar wurde. Bei 6 der erscheinungsfrei gebliebenen Probanden konnte ein Virusnachweis geführt werden. Die gleiche Arbeitsgruppe um HenLe [286] befaßte sich auch mit Einsatz derartiger Infektionen in der Prophylaxe der Mumpserkrankung. Zuvor hatten ENDERS u. Mitarb. [181] auf Grund virologisch-immunologischer Untersuchungen nachweisen können, daß nach stumm verlaufenden Mumpsinfektionen gleiche Antikörpertiteranstiege wie nach manifesten Erkrankungen eintreten konnten. Von Overman u. Kilham [51\%] wurde bei über 9 Tagen alten Goldhamstern nach Inoculation des hamsteradaptierten IC-Mumpsvirus ein Mumps-Antikörperanstieg im Serum der Tiere ohne Erkrankung berichtet; es ist dabei besonders bemerkenswert, daß Tiere unterhalb dieses Alters bei gleichen Versuchsbedingungen an einer Encephalitis eingingen. Eine gleiche Altersabhängigkeit beobachtete OvERMaN [516] auch bei der Säuglingsmaus, wohingegen KilHam u. Murphy [373] eine erwiesene inapparente Säuglingsmaus-Infektion mit Gewebekulturpassiertem Mumpsvirus mitteilten.

Wie schon erwähnt, hat Mathis [462] 1938 in seiner Monographie über das Gelbfieber die klinisch stummen Infektionen als Schulbeispiel für eine inapparente Infektion hingestellt. Das bis auf spärliche Zweifelsfälle erwiesenes Ausbleiben einer apparent pathogenen Wirkung des D-17Stammes dieses Virus [685] auf den Menschen und Affen [159; 201; 321; 684; u. a. m.] bei Anregung einer Antikörperbildung und Schaffung einer protektiven Immunität war die Veranlassung zur Verwendung dieser Variante als Impfstoff.

Einer ähnlichen Situation stehen wir bei der Anwendung eines - allerdings auf gänzlich anderem Wege geschaffenen - ,Virus fixe“ des Tollwut-Virus gegenüber (zusammenfassend bei Rooyen u. RHodes [582]). Ein immunisatorischer Schutzeffekt ist auch beim Meerschweinchen, beim Hund und beim Affen nach experimenteller, klinisch erscheinungsfrei bleibender, bzw. abortiv verlaufender Infektion mit dem Ei-adaptierten FLury-Stamm [393, 394] des Rabies-Virus nachgewiesen [390_392; 570]. Und vermutlich läuft auch bei symptomlosen Fällen der ,,neuroinfection autostérilisable" von Hühnern und Tauben nach intracerebraler Inoculation des ,Straßenvirus“ der Tollwut [568, 569] eine inapparente Infektion ab.

Die Poliomyelitisforschung bietet - in neuester Zeit ebenfalls häufig mit der Zielsetzung eines protektiven Effektes - gleichartige Beispiele für inapparente Infektionsverläufe. Hierzu zählen außer vorausgegangenen Affenversuchen mit Nachweis einer Immunität nach experimentell induzierter inapparenter Infektion [z. B. $86,88,89 ; 125 ; 313 ; 318 ; 439$; 
476, 47\%; 600; u. a. m.] in erster Linie die am Menschen durchgeführten oralen Infektionsversuche mit Nager-adaptierten, für den Menschen apparent avirulenten Poliomyelitisviren [395-400], sowie die ebenfalls beim Menschen und Affen, teils oral teils auch durch i.m. Injektion vorgenommenen Infektions-Experimente [596-598] mit apparent apathogenen Varianten der drei Poliomyelitis-Virustypen, welche SabIN, Hennessen u. Winsser [598] durch schnelle Passagen auf Kulturen nichtneuraler Gewebe erzeugen konnten. In jenen Versuchen wurden hohe Titeranstiege homologer Antikörper beobachtet. Allerdings sind die Bedingungen zum Beweis einer inapparenten Infektion im engsten Sinne bei diesen Versuchen insofern nicht erfüllt, als sowohl SABIN wie auch KoprowsKI u. Mitarb. darauf verweisen, daß der Nachweis einer Virämie bei den Probanden bisher nicht gelungen sei. Eine symptomlose Übertragung von Poliomyelitis-Virus auf den Nager Meriones Shawi wird durch BLANC u. MarTIN [78] berichtet. Diese Autoren stellen eine allerdings auf Grund ihrer Angaben nicht bewiesene Adaptierung des Poliomyelitis-Virus auf Kaninchen zur Diskussion; die einzige Reaktion infizierter Versuchstiere bestehe dabei in einem kurzdauernden Temperaturanstieg. LEvINsoN u. Mitarb. [429] berichten über den Anstieg neutralisierender typenspezifischer Antikörper gegen alle drei Poliomyelitis-Virustypen im Kaninchenserum nach wiederholter i.m. oder i.v. Injektion von VirusGewebekultur-Suspensionen; die Tiere zeigten keine Krankheitssymptome. Die gleiche Beobachtung wurde von JezIERski [334] bei Versuchen mit dem Esel gemacht. Die Beurteilung derartiger Reaktionen im Rahmen einer Besprechung der inapparenten Infektion wirft aber wiederum jene Fragen auf, welche die Abgrenzung einer Immunkörperbildung als Ausdruck einer echten Infektion einerseits, oder lediglich eines von dem infektiösen Prozesses grundsätzlich unabhängigen Antigeneffektes andererseits betreffen; die Fähigkeit des Kaninchens, auf den Antigenreiz inaktivierten Poliomyelitis-Virus mit der Bildung neutralisierender Antikörper zu reagieren, ist bekannt und $\mathrm{u}$. a. von Keller u. SAUthoff [360] sowie - wie schon erwähnt - von LeviNsoN u. Mitarb. bewiesen worden. Die mitgeteilten Beobachtungen haben für das Postulat der inapparenten Infektion daher keine überzeugende Beweiskraft.

Einen sicheren Nachweis für das Vorliegen einer inapparenten Infektion haben Kraft u. Melnick [404] sowie Melnick u. Kaplan [475] durch die Fütterung von Schimpansen mit Coxsackie-Virusstämmen erbracht. Sie konnten ohne jeglichen pathogenen Effekt dieser Agentien die Virämie, die Virusmultiplikation im Organismus, den Anstieg neutralisierender Antikörper und den über Monate anhaltenden Immunitätsschutz (Ausbleiben einer Virämie und Verkürzung der Virusausscheidungsdauer nach erneuter Infektion) nachweisen. 
Über ein aufschlußreiches Massenexperiment - wobei die Existenz einer inapparenten Infektion durch unbeabsichtigte Steigerung einiger Fälle in das Stadium der manifesten Erkrankung gezeigt wurde - berichteten Sutton u. Brocke [643]. 327 Personen waren durch insgesamt 1174 Inoculationen mit einer als Menschen-apathogen deklarierten, immunologisch hoch wirksamen Vaccine von Venezuelanischer Pferdeencephalitis geimpft worden. Es traten bei dieser Aktion 14 klinische Encephalitisfälle auf; hierbei konnte - entgegen den bis dahin verbindlichen Angaben, daß bei einer solchen aktiven Immunisierung eine Virämie nie nachzuweisen sei - in 7 von 10 dieser Erkrankungen das Virus zwischen dem 1. und 5. Tag p. inocul. aus dem Blut isoliert werden. Eine Infektionsmöglichkeit außer durch die Vaccinierung wurde für alle Fälle verneint. Auch im Gefolge einer Infektion von Rhesusaffen mit dem Virus der Pferde-Encephalitis ist ein stummer Kontakt durch den vom 4. Tag nach der Infektion nachweisbaren Gehalt des Serums an neutralisierenden Antikörpern dokumentiert [324]. Einen weiteren Modellbeweis für eine inapparente Infektion liefern Morris, O'Connor u. SMadeL [490]. Bei Cynomolgusaffen konnte nach i.v. Inoculation des russischen Frühjahr-Sommer-Encephalitis-Virus eine mehrtägige beträchtliche Virämie und nach etwa 1 Woche ein Anstieg spezifischer Antikörper festgestellt werden, ohne daß die Tiere krankhafte Erscheinungen gezeigt hätten.

Die inapparente Infektion mit einem encephalitogenen Virus ist ebenfalls durch Untersuchungen von WHITNEY [\%40] bestätigt; nach den Angaben dieses Autors verläuft eine Infektion frisch isolierter Stämme von Choriomeningitis-Virus bei Säuglingsmäusen ohne Krankheitszeichen und hinterläßt einen Immunitätsschutz, während ausgewachsene, nicht latent durchseuchte Mäuse stets hochempfindlich im Sinne der Ausbildung einer manifesten Erkrankung sind. Über ein gleichsinnig altersabhängiges Verhalten bei einem Kontakt zwischen der Maus und dem Lansing-Poliomyelitis-Virus berichtete SABIN [595].

Von dem Phänomen unterschiedlicher pathogener Effektivität eines encephalitogenen Virus auf verschiedene Wirtsspecies macht auch die Veterinärmedizin bei aktiven Immunisierungsmaßnahmen Gebrauch. So propagieren Alexander u. DU ToIt [10] - um nur wenige Beispiele zu nennen - den neurotropen Maus- und Meerschweinchen-adaptierten Virusstamm der Pferde-Encephalitis als Vaccine für Pferde und Maulesel; und SmrthburN [633] benutzt das gleiche Verfahren für die experimentelle Vaccinierung mit Rifttalfieber-Virus. Auch eine Immunisierung gegen die Newcastle-Krankheit des Geflügels [296] beruht auf diesem Prinzip. Bei diesen Maßnahmen ist allerdings im Auge zu behalten, daß auch hier - wie schon mehrfach gesagt - die echte Infektion bewiesen und von der Antigenwirkung allein abgegrenzt werden muß. Eine Nutzung der inapparenten Infektion für Vaccinierungszwecke liegt 
aber zweifellos bei Simultan-Impfungsverfahren gegen virale Tierseuchen, wie z. B. bei der Maul- und Klauenseuchenimpfung [\%25] vor.

Für die Virusinfektionen des Respirationstraktes können auch Beispiele inapparenter Infektionen genannt werden. Nach den Untersuchungen von Hirst [294, 295], von WaNc [\%27], von Matsumoto u. Mitarb. [463] sowie von FRIEdewald u. Hook [214] werden Ei- oder Frettchenpassierte, also nicht Maus- oder Hamster-adaptierte Grippe-Viren in der Maus- oder Hamsterlunge ohne jegliche Läsion multipliziert. Die gewebliche Irritation und der pathogene Effekt treten dann in der 4 . bis 6 . bzw. nach Matsumoto u. Mitarb. bei dem CAM-2 M-Stamm des A-Typs in der 9. fortlaufenden Lungenpassage abrupt auf. Allerdings fehlen für den sicheren Beweisantritt der inapparenten Infektion hier ausreichende Immunitätsprüfungen.

Bisher sind wir davon ausgegangen, daß die jeweilige Infektion durch den willkürlichen Eingriff der Inoculation sicherstand. Die Aufdeckung asymptomatischer natürlicher Infektionen, bzw. deren Annahme auf Grund einer vermehrten Exposition bei Nachweis einer immunologischen Reaktion der exponierten Individuen vermehrt den Einblick in die Fragen der inapparenten Infektion. Auch für diese Gegebenheiten seien einige Beispiele aufgeführt.

Eine Isolierung von Coxsackie-Viren und der Nachweis von Antikörpern gegen diese Agentien gelingt nach den Mitteilungen zahlreicher Autoren $[54 ; 142,143,145 ; 338 ; 364,471 ; 662 ; 720 a$; 726; u. a. m.] bei gesunden Personen und besonders in der Umgebung von entsprechenden Erkrankungsfällen häufig; DALLdorF hat diese Tatsache mit dem Ausspruch apostrophiert, daß ,,we are in the anomalous position of having discovered the cause of a disease before discovering the disease"; eine gleichsinnige Beurteilung, mit dem Schwergewicht auf der klinischen Bedeutung äußern WINDORFER u. Schricker [\%48, 749], wenn sie mit Nachdruck auf ,die rudimentären Krankheitsbilder in der Umgebung typisch Erkrankter" hinweisen.

Die gleiche Situation liegt bei Infektionen mit dem Poliomyelitis-Virus vor. Wenn hier die These vom Überwiegen des Infektionsablaufes ohne typische paralytische Krankheit auch keineswegs neu ist, so haben doch jüngere Untersuchungen von Bodian u. Paffenbarger [91], Brown u. Ainslie [108]; Howe u. Bodian [316]; Lépine [421]; McClure [466], Malherbe [456], Melnick u. Ledinko [4\%7], Pavilanis u. Frappier [525], Paul, Melnick u. Riordan [522]. Ramos-Alvarez u. Sabin [559], Siegel, Greenberg u. Stone [625], Trask, Paul u. Vignec [694], Wenner u. Mitarb. [736, 739] und vor allem von Honig u. Mitarb. [304] sowie von Horstmann u. Mitarb. [310-312] ein ausgezeichnetes Beweismaterial für die Existenz unterschwelliger Poliomyelitisvirus-Infektionen 
vorgelegt. HonIg u. Mitarb. [304] konnten bei 136 völlig gesunden Kindern in wiederholten, über 29 Monate verteilten virologischen Kontrollen von 1558 Stuhlproben 77 Virusstämme isolieren, davon $44 \%$ sogenannte „Orphanviren“, 37\% Coxsackie- und 19\% Poliomyelitisviren; der Beweis eines biologischen Reaktionskontaktes mit dem jeweiligen Erreger wurde bei einer Anzahl von diesen Individuen durch SerumantikörperAnstiege erbracht. Derartige inapparente Infektionen schienen häufiger in sozial schlechterem Milieu $(8,3 \%)$ gegenüber gehobenen Schichten $(3,1 \%)$. Horstmann, McCollum u. Mascola [312] fanden bei 91 Individuen, welche Kontakt mit Poliomyelitiskranken gehabt hatten, 21 virologisch und immunologisch gesicherte inapparente Infektionen und 7 aparalytische Krankheitsverläufe. MaLHeRbe [456] stellte bei 46 Kindern aus der Umgebung eines letalen Leon-Poliomyelitisfalles in 53\% eine kurzdauernde und bei 8,1\% eine längerdauernde (über drei Wochen anhaltende) Ausscheidung dieser Erreger fest. Es ergibt sich zwanglos aus diesen letztgenannten Befunden, daß die bei der Mehrzahl der erfaßten Infizierten nachgewiesene Autosterilisation als Zeichen einer Immunität und somit als Ausweis für eine inapparente Infektion gefolgert werden kann. Ohne auf die stark divergierenden Angaben über das Verhältnis der inapparenten zu den apparenten Poliomyelitis-Virusinfektionen eingehen $\mathrm{zu}$ wollen, das nach Horstuans [308] zwischen 8:1 und 100:1, dagegen nach Melnick u. Ledinko [47\%] sowie nach KelleR [358] in jüngeren Altersstufen und bei sozial niedrigstehenden Bevölkerungsgruppen bis 1000:1 betragen kann, darf das Resumé über die Existenz inapparenter Poliomyelitis-Virusinfektionen mit LÉPINE $u$. BoYER [422] wie folgt zusammengefaßt werden : ,La ,Polio-infection“ est beaucoup plus répandue que la ,polio-maladie‘ ce qui signifie que dans un très grand nombre de cas, l'infection par le virus est sans traduction clinique. Or, tous les sujets qui sont porteurs sains de virus, tous ceux qui présentent des formes d'infections inapparentes représentent l'immense majorité." Mitgeteilte Beobachtungen, daß in der Umgebung menschlicher paralytischer Poliomyelitisfälle auch rudimentäre zentralnervöse Störungen bei Haustieren in Erscheinung traten $[33 ; 206]$, beanspruchen innerhalb der Problematik inapparenter Infektionen ebenfalls Berücksichtigung; dabei ist es in diesem Zusammenhang durchaus gleichgültig, ob es sich um echte Poliomyelitisvirus-Infektionen oder um poliomyelitisartige Syndrome nach Infektion mit anderen neurotropen Viren gehandelt hatte. Als Beispiel hierfür seien die Beobachtungen von Pellissier u. Trinquier [532-534] während einer kleinen Poliomyelitisepidemie in Brazzaville genannt; das hierbei isolierte und für die Erkrankungen verantwortlich gemachte ,,virus encéphalomyélitique de Brazzaville“ wurde auch aus Patientenblut einer ,,minor illness" isoliert und erst später als Poliomyelitisvirus-Variante identifiziert. 
Aus einer epidemiebedingten Nachprüfung der Antikörper gegen Meningoencephalitis-Virus an 182 polnischen Waldarbeitern zogen PosPIŠILová, SNítillová u. DĚdková [553] den Schluß, daß neben 20 klinisch erkrankten Probanden 21 gesunde Personen mit erwiesenen Serum-Antikörpern eine abortive Form der Krankheit durchgemacht haben mußten.

Die seuchenhygienische Bedeutung inapparenter Tierinfektionen mit encephalitogenen Viren wird unter anderem durch den Nachweis von Rabies-Virus in scheinbar gesunden Vampyren hervorgehoben; die fatale Rolle dieser Tiere als Überträger der Tollwut ist schon lange besonders von Beobachtungen auf der Insel Trinidad bekannt [582]. Sie wird unterstrichen durch neuerliche Mitteilungen von Kougr [402] sowie von Venters u. Mitarb. [\%11]. Veranlaßt durch Bißverletzungen zweier Menschen durch gelbe Florida-Vampyre (Desypterus floridanus) konnten sowohl bei den beißenden Tieren die Rabies-Virusinfektion nachgewiesen als auch von 208 apparent normalen Vampyren der Gegend sechsmal das Rabies-Virus isoliert werden; die Tatsache, daß nicht bei allen diesen Tieren Negrikörperchen gefunden und Antikörper nachgewiesen worden waren, läßt allerdings die Möglichkeit offen, daß es sich nicht um eine inapparente Infektion im strengen Sinne, sondern um Inkubationszustände gehandelt hat. Bei derartigen Beobachtungen meldet sich auch wieder die Frage nach der Möglichkeit eines Trägertums. Darüber wird im weiteren noch zu sprechen sein.

Die Ermittlung eines stummen, aber doch echten Reaktionskontaktes mit dem Mumps-Virus teilen unter anderen Esser-Trimmberger u. KцöNE [185] mit. Von 152 Normalseren, welche wahllos zusammengestellt worden waren, zeigten $14 \%$ in der Komplementbindungsreaktion einen Titer von über 1:8, von 144 in Krankenhäusern beschäftigten Personen hatten $30 \%$ den gleichen Titer, von 44 Personen, welche in einem Lager in engem Kontakt mit Mumpskranken lebten, wurden in $43 \%$ diese Titerhöhe bestimmt. Nach LIPPELT u. MÜLLER [441, 442] ist die Übertragung dieser offensichtlich auf nordamerikanische Verhältnisse mit sehr differierender Immunitätslage zwischen Stadt- und Landbevölkerung bezogenen Situation für deutsche Verhältnisse nicht erlaubt, da hier die Gesamtbevölkerung im mittleren Alter von 28 Jahren in etwa 80\% Antikörper gegen das Mumpsvirus aufweist.

Auch bei Q-Fieber-exponierten Personen liefert die Bestimmung der Titer komplementbindender Antikörper gleichsinnige Ergebnisse [283; 503]; außer den Erkrankten dieser Untersuchungsgruppen konnte auch hier bei erscheinungsfrei gebliebenen Kontaktpersonen eine auf die vorausgegangene inapparente Infektion hinweisende Antikörper-Titerhöhe ermittelt werden.

Wenn aus der kürzlich veröffentlichten Zusammenstellung über das Wolhynische Fieber [483] eine gleichsinnige Beurteilung nicht sicher 
ableitbar ist, so kann doch aus der Abhandlung über die Epidemiologie des Wolhynischen Fiebers von Kostrezewski [401] entnommen werden, daß es auch bei der Infektion mit $R$. wolhynica symptomarme bzw. symptomlose Fälle mit einer beträchtlichen Virämie gibt.

Schließlich verweisen wir auf Mitteilungen über epidemiologische Studien von Hilleman u. Mitarb. [291, 292], welche an kasernierten jungen Männern während einer Häufung von akuten Erkrankungen der Luftwege vorgenommen worden waren. Auch hier sollen nach Angaben der Autoren bis zu 50\% der Infektionen mit dem als Ursache ermittelten Virus RI-67 der sogenannten ARD-(akute respiratory disease)-Viren entweder sehr mild oder inapparent verlaufen sein. Die bisher gewonnenen Kenntnisse über diese erst in jüngster Zeit präzise abgegrenzte Virusgruppe sind kürzlich ausführlich von LIPPELT [439a] referiert worden. Aus dieser Darstellung ist einerseits die weite Verbreitung jener Erreger - unter welchen vor allem die APC-(adenoidal-pharyngeal-conjunctival) und AD-(adenoid degenerating)-Viren zu nennen sind - zu erkennen. Die gegenüber entsprechenden klinischen Erkrankungen ungleich häufigeren Nachweise spezifischer Antikörper erlauben außerdem den Rückschluß auf zahlreiche inapparente Infektionsverläufe.

Endlich soll - unter Vorgriff auf die Besprechung der möglichen Auswirkungen einer inapparenten Infektion, - auf die Mitteilung eines humanen Erkrankungsfalles an akuter hämolytischer Anämie mit Autohämagglutination verwiesen werden [484], wobei nach Ablauf der akuten Krankheitsphase das Newcastle-disease-Virus aus dem Blut isoliert werden konnte. Auch hier kann man vorerst mangels Vorliegen der für gelegentliche menschliche Newcastle-disease-Virusinfektionen als charakteristisch beschriebenen Schleimhautaffektionen der oberen Luftwege und der Konjunktiven eine inapparente Infektion diskutieren allerdings mit einer fatalen Sekundärwirkung. Auch bei CoxsackieVirusinfektionen sind solche Auswirkungen beschrieben [64, 67].

\section{Der Rückschluß von der Auswirkung auf die vorangegangene Infektion}

Wir haben bislang versucht, die Existenz einer inapparenten Infektion von der erwiesenen oder vermutbaren Infektion auf ihren Effekt im einzelnen Individuum abzuleiten. Im weiteren soll davon ausgegangen werden, daß es für die hier besprochenen Infektionen erlaubt ist, aus dem Erwerb spezifischer Antikörper ohne vorangegangene manifeste Erkrankung trotz fehlenden Nachweises der Infektion selbst gleiche Schlußfolgerungen zu ziehen.

So berichten Batwell, Denel, Matumoto u. Sabin [49] über Bestimmungen von Antikörpertitern gegen Japanische B-Encephalitis im 
Serum von früher nicht erkrankten Menschen und Haustieren in Korea und Japan; ein Antikörpernachweis von z. B. 78\% in der Altersgruppe der 60 jährigen Menschen wird von den Autoren als Hinweis für zahlreiche inapparente Infektionen mit diesem Erreger angesehen. Dieser Wert nähert sich den Ergebnissen gleichartiger Untersuchungen [552] mit 210 Seren aus Malaya (74\% Antikörperfunde gegen Japan. B-Encephalitis) und Borneo (67\% Antikörperfunde). Die Immunitätsstudien, welche Smithburn u. Mahaffy $[634,635]$ in Ostafrika durch Neutralisationstests gegen zahlreiche dort isolierte ,Arthropod-borne“Viren (Bwambafieber-, Ntaya-, Zika-, Uganda S-, West Nile-, Bunyamwera- u. a. m. -viren) vorgenommen haben, lassen auch dort unter den Einwohnern eine ganz beträchtliche Durchseuchung erkennen, wobei auf einen ansehnlichen Anteil inapparenter Infektionsverläufe geschlossen werden kann.

Die Poliomyelitisforschung bietet analoge Ergebnisse in großer Zahl. Aus der Fülle der Berichte sei nur auf die Antikörpernachweise von Aycock [35], Clark u. Rhodes [131], Fallon [186], Goldblum u. Melnick [242], Hammon u. Sather [266], Hennessen u. Sauthoff [287], Horstmann u. Kraft [314], Keller u. Viveld [365], Lennartz u. Müller [420a], Melnick u. Ledinko [477], Paul u. Mitarb. [521, 522, 524], du Pan [518a], Pavilanis u. Lépine [526] sowie von Pintér [546] verwiesen. Úberall bestätigt sich das Phänomen, daß der Vergleich eines Antikörpergehaltes menschlicher Seren mit den durch eine Erkrankung markierten Infektionen eine so erhebliche Diskrepanz zugunsten der Antikörpernachweise ergibt, daß eine Erklärung hierfür nur durch Heranziehen häufiger inapparenter Infektionen möglich ist. In warmen und hygienisch vernachlässigten Regionen tritt dies besonders deutlich hervor; die Diskussion hierüber ist von einem Teil der genannten Autoren und anderen bereits so ausgiebig geführt worden, daß wir vorerst darüber hinweggehen können.

Auch die Mumps-Virusinfektionen sind nach Ausweis immunologischer Untersuchungen häufiger als dies von den Mumpserkrankungen abgeleitet werden kann $[255 ; 284 ; 364 ; 460 ; 523 ; 627]$; bei der Annahme eines Antikörpernachweises bei $80-90 \%$ gedrängt lebender Bevölkerungsgruppen müßte ein Teil der Mumpsinfektionen inapparent verlaufen. Aber auch im Falle einer geringeren Durchseuchung kann diese Annahme aufrecht erhalten werden; z. B. ermittelten BASHE u. Mitarb. [48] in dünnbesiedelten Gebieten Alaskas bei 126 Probanden ohne Mumpsanamnese in 23,8\% einen positiven Nachweis neutralisierender Mumps-Antikörper im Serum.

Auf immunologische Untersuchungsergebnisse von BELL, BECK u. Huebner [57], ferner von MeIkLejohn u. Lenette [473] sowie von Sulkin u. STtRauss [642] über Antikörper-Titeranstiege gegen $C$. burneti 
ohne vorangegangene Erkrankung darf in diesem Zusammenhang ebenfalls verwiesen werden. Auch hieraus ist zu entnehmen, daß inapparente Q-Fieber-Infektionen durchaus nicht selten sind; es dürfte dabei epidemiologisch nicht ohne Bedeutung sein, daß solche Befunde (Sulkiv u. Strauss) gerade bei Arbeitern in der Fleischverarbeitungsindustrie erhoben werden konnten.

Hillemann, Werner u. Gauld [293] veröffentlichten 1954 eine Studie über den Nachweis von Antikörpern gegen Viren der Influenzagruppen A, B und C in den Seren von gesunden Kindern und Erwachsenen der USA-Bevölkerung. Diese mittels des HämagglutinationsHemmungstests durchgeführten und die Jahre 1943-1951 erfassenden Untersuchungen lassen eine Korrelation zwischen zeitlichen Morbiditätshäufungen und Antikörper-Titeranstiegen gegen A- und B-Grippeviren erkennen. Gänzlich anders lag die Situation dagegen bei den Testungen des hämagglutinations-hemmenden Vermögens von 296 Erwachsenenseren gegenüber dem 1233-Stamm [6\%\%, 6\%8] der C-Influenzaviren. Es wurde während der einbezogenen 9 Jahre eine ziemlich gleichbleibende Antikörpertiterhöhe um 1:80-1:320 (Serumverdünnung) nachgewiesen; gleichhohe Titer fanden sich bei Kindern von über 3 Jahren - im Gegensatz zu erheblich geringeren Antikörpernachweisen gegen A- oder B-Grippeviren in der gleichen Altersklasse. Setzt man diese Befunde in Vergleich zu den Beobachtungen von Francis, Quilligan u. Minuse [205] sowie von GERBER u. Mitarb. [233], nach welchen InfluenzaC-Virusinfektionen klinisch gewöhnlich ziemlich bland sind, so ist zumindest für die Influenza-C-Viren zu folgern, daß humane Infektionen überwiegend inapparent verlaufen.

Das Phänomen einer nur serologisch ermittelbaren, klinisch aber völlig stummen Auseinandersetzung des Menschen mit epizootischen Viren ist auf Grund des Nachweises von komplementbindenden Antikörpern gegen das Theilersche Mäuseencephalomyelitisvirus und gegen Viren der Col.-SK-Gruppe in menschlichen Seren von KELLER u. VIVELL [364] sowie von Seligmans u. Jungeblut [608], von Enright u. SADLER [182] gegen das Virus der bovinen Encephalomyelitis, sowie von Stanley, Dorman u. Ponsford [663] für ein von diesen Autoren kürzlich beschriebenes mäusepathogenes Agens, das HEV (Hepatoencephalomyelitis-virus) mitgeteilt worden.

Die angeführten Beispiele lassen erkennen, von welcher Bedeutung derartige immunologische Prüfungen einer Durchseuchung und ihr Vergleich mit den jeweiligen Morbiditätsindices für eine Abschätzung sowohl bevölkerungshygienischer Situationen als auch epidemiologischer Beurteilungen sind; der Ausbau einer solchen ,,serologischen Epidemiologie" [364; 523] verschafft gleichzeitig der Klinik Anhaltspunkte für die Bewertung von infektiösen Prozessen im allgemeinen, und weist im 
besonderen auf die eventuelle Existenz atypischer, abortiver latenter oder inapparenter Verläufe hin.

Die Möglichkeit eines Rückschlusses von geweblichen Veränderungen auf eine im übrigen symptomlos gebliebene Virusinfektion ist beim Menschen an Zufallsbefunde gebunden. Für eine Beweisführung inapparenter Infektionen sind kasuistische Beobachtungen dieser Art außerdem schon deshalb von zweifelhaftem Wert, weil sie einen noch nicht abgeschlossenen Prozeß ausweisen und damit gar keine Aussage darüber erlauben, ob es sich wirklich um eine inapparente Infektion unserer Definition handelt. So sind z. B. die von Finkeldey [197] und WARTHIN [730] beschriebenen mesenchymalen Riesenzellen innerhalb des lymphatischen Gewebes ,,anginöser“ Tonsillen und in der Pharyngealschleimhaut, sowie die von Davidsohn u. Mora [149], Fischer [198], Herzberg [290] und Schultze [661] in der Appendix und von HathaWAY [272] und WEGELIN [\%31] in sonstigen lymphatischen Geweben nachgewiesenen gleichartigen Zellen zum Zeitpunkt ihres Auftretens zweifellos spezifische Korrelate von klinisch noch nicht erkennbaren Masernerkrankungen, die klinischen Symptome folgen aber bei dieser, einen extrem hohen Kontagionsindex aufweisenden Infektion mit einer an Sicherheit grenzenden Wahrscheinlichkeit noch nach. Auch mit Einschlußkörperchen-Nachweisen kann die Annahme einer inapparenten Infektion nicht begründet werden. Ihr Vorhandensein besagt im allgemeinen ebenfalls, daß der Infektionsprozeß noch nicht abgeschlossen ist; damit muß die Frage einer Apparenz oder Inapparenz natürlich offen bleiben. Und schließlich erlauben narbige Gewebsbilder abgeheilter Läsionen eher Vermutungen als beweiskräftige Rückschlüsse auf die zu unterstellenden Noxen. Einblicke in die geweblichen Veränderungen bei inapparenten Virusinfektionen sind deshalb im wesentlichen auf experimentelle Studien über morphologische Korrelate unterschwelliger Virusinfektionen beschränkt. Sie werden ergänzt durch Ergebnisse weniger systematischer Untersuchungen über das Ausmaß histologischer Reaktionen im Verlaufe von infektiösen Prozessen, bei welchen ein Vergleich der manifesten Krankheitserscheinungen mit dem nachgewiesenermaßen viel stärker ausgebreiteten geweblichen Schaden zu dem zwingenden Schluß führt, daß ein beträchtlicher Teil der geweblichen Irritationen symptomlos bestanden haben mußte; hierzu zählen die eindrucks- und verdienstvollen histologischen Poliomyelitisstudien von Baker, Cornwell, Matzke u. Mitarb. [39-41, 110, 464, 465]. Solche Fragen und ihre Beantwortungsmöglichkeiten sollen jedoch gesondert abgehandelt werden. So bleiben für eine Erkennung inapparenter Virusinfektionen mittels morphologischer Methoden im wesentlichen nur die Auswertungen blutmorphologischer Untersuchungen übrig. Zahlreiche 
Viruserkrankungen (z. B. Masern, Pocken, Mumps, Virusencephalitiden, Poliomyelitis, Gelbfieber, Maul- und Klauenseuche, Psittakose, Grippe) gehen mit einer ausgeprägten lymphoid-zelligen und plasmacytären Reaktion einher. Der Nachweis von lymphoiden Reizformen (,Virocyten“, SIEDE [622]; u. a. m.) im Hämatogramm solcherart Infizierter ist ebenso wie das Auftreten der charakteristischen Zellformen bei Auseinandersetzungen mit ,lymphotropen“"Viren (akute infektiöse Lymphocytose, Rubeolen, Mononucleose, Hepatitis epidemica) in den allerseltensten Situationen, - und auch nur in Verbindung mit anderen Hinweisen, - ein verwendbares Argument zur Beweisführung einer inapparenten Infektion. Am ehesten gilt dies noch für die infektiöse Lymphocytose. Eine in diesem Sinne verwendbare Beobachtung sei hier angeführt: Von Scalettar, Maisel u. Bramson [605] wurde in Zeiteinheit mit der manifesten Erkrankung eines 3jährigen Waisenhauspflegekindes an akuter infektiöser Lymphocytose mittels Umgebungsuntersuchungen an 154 Kindern dieser Anstalt in 16 Fällen eine klinisch asymptomatische Lymphocytose (Werte bis $80 \%$ ) über etwa 5 Wochen Dauer nachgewiesen. Dem Kinderarzt ist andererseits die extreme Lymphopenie bei virus-bedingten kindlichen ,Infekten“ der oberen Luftwege und die in der Rekonvaleszenz nachfolgende Lymphocytose ebenso geläufig wie Beobachtungen gleicher Art bei scheinbar nicht-affizierten Kindern in der Umgebung solcher Fälle.

Es muß aber noch einmal betont werden, daß solche Befunde zwar einmal einen Fingerzeig für den Ablauf einer inapparenten Virusinfektion, nicht aber für deren ätiologische Beweissicherung liefern können; diese setzt die Erfüllung der Koch-Henle-Riverschen Postulate voraus. Außerdem ist bei dem Augenblicksbild einer geweblichen Irritation jede Beurteilung über die heuristische Feststellung hinaus unangebracht. Erst die Klärung der Ätiologie und die Übersicht über den Prozeßablauf, bzw. die retrospektive Auswertung gestatten die Einordnung solcher Geschehen in der Stufenfolge von dem inapparenten über den abortiven bis zum klinisch manifesten Prozeß.

\section{Die Abgrenzung der inapparenten Infektion gegen andere Formen unterschwellig verlaufender Virusinfektionen}

Ein Ưberblick über die Phänomenologie zahlreicher Virusinfektionen setzt unsere Bemühungen um eine Abgrenzung der ,inapparenten Infektion" dem wiederkehrenden Einwand aus, daß eine Anzahl von Kontakten empfänglicher Wirte mit einer Reihe von Viren zwar durchaus ohne Krankheitserscheinungen verlaufen kann, dem Begriff der ,,inapparenten Infektion" jedoch nicht unterzuordnen ist. Zunächst soll einmal auf die Frage der Berührungspunkte zwischen Trägertum und 
inapparenter Infektion eingegangen werden. Nach DoenR [166] ist ein Träger - ganz allgemein gesprochen — ,ein Individuum, welches in seinem Organismus krankheitserregende Keime beherbergt, ohne daß dieser Zustand in pathologischen Erscheinungen zum Ausdruck kommt"; und DoERr fährt fort: ,,in dieser weitesten Fassung deckt sich der Begriff des Trägertums mit der Definition der latenten Infektion." Nun haben wir bereits gezeigt, daß die ,,inapparente Infektion“ im Sinne Nicolles in den Latenzbegriff von DoerR innerhalb der „Latenz als cyclischer Prozeß " Aufnahme finden kann. Damit wäre grundsätzlich die Verbindung der beiden Begriffe miteinander aufgezeigt. Die Divergenz in der Bedeutung wird aber doch offensichtlich, wenn DoERR in der Weiterverfolgung seiner Begriffsbildung das Trägertum als ,,den latenten Infekt in seiner epidemiologischen Auswirkung“ bezeichnet; die ,inapparente Infektion" muß dagegen die auf den einzelnen befallenen Organismus bezogene ,maladie aigue“ bleiben. Dies sind zwei voneinander unterscheidbare, wenn auch nicht notwendigerweise in einem und demselben Individuum zu trennende Situationen. Die Trennung ergibt sich aber in der Bewertung eines solchen Zustandes dennoch, wenn wir berücksichtigen, daß es gerade cyclische, zur Autosterilisation hinstrebende Virusinfektionen sind, deren unterschwellige Verlaufsformen die ,,inapparenten Infektionen" repräsentieren. Am speziellen Beispiel der Poliomyelitis wollen wir versuchen, die allgemeinen und grundsätzlichen Gesichtspunkte einer solchen Situation zu umreißen. Zunächst ist es ratsam, sich bei allen Verlautbarungen über das Vorliegen eines ,,Trägertums" davon zu überzeugen, wovon der Berichterstatter de facto spricht. Wenn in der älteren Poliomyelitisliteratur Wickman [741], Flexner u. Mitarb. [200], Kling u. Petterson [381], Wurtz [752] auf die Bedeutung von „Keimträgern“" hinweisen, so läßt eine Durchsicht dieser Berichte erkennen, daß es sich dabei um gesunde, aber - durch Virusnachweis im Nasopharynx erwiesen - infizierte Personen aus dem Lebenskreis eines an akuter klinischer Poliomyelitis erkrankten Menschen handelte. In diesen Berichten wird gar nicht der Versuch gemacht, einen Trägerzustand und eine subklinisch ablaufende echte Auseinandersetzung mit dem Erreger zu differenzieren. Oder aber es wird eine solche Trennung einfach nicht für notwendig erachtet, wie dies aus den Worten von WuRTz hervorgeht, welcher schreibt, daß die Infektionsrate größer sei als die Erkrankungsrate und: aus diesem Grunde ,,unsuspected carriers are many". Eine Unsicherheit der begrifflichen Vorstellung spiegelt sich auch noch in Berichten von LÉpINE u. Mitarb. [421, 425]. LÉpINE berichtet über einen ,,porteur sain“, welcher Kontakt mit einem Poliomyelitiskranken gehabt hatte und nun nachweislich über 123 Tage das Virus - wenn auch mit fortschreitender Zeit in immer kleineren Mengen

- im Stuhl beherbergte; der mitgeteilte Befund neutralisierender 
Antikörper im Serum dieses Menschen weist aus, daß es sich in diesem Falle nicht um einen ,,porteur sain“, sondern um einen ,,porteur convalescent" gehandelt haben muß, und daß hier eine unterschwellige Infektion abgelaufen ist, deren Auswirkungen zweifellos in der abklingenden Virusausscheidung infolge einer Tendenz zur Autosterilisation erfaßbar wurden. In solchen Fällen wird die Entscheidung darüber, ob es sich noch um eine abklingende inapparente Infektion handele, von der Situation des Klinikers und der des Parasitologen verschieden sein; offenkundig wird aber an diesem Beispiel die sinnvolle Ordnung, in welche DoERR die Phänomene aus der Schau des Mikrobiologen gefügt hat. Sie werden den klinischen Belangen allerdings nicht gänzlich gerecht. Wenn nämlich bei einem Träger in der Definition DoERRs pathologische Erscheinungen ,nicht zum Ausdruck kommen“, so schließt dies eine unterschwellige Existenz von deren Substrat nicht aus. Hierauf aber gründet die ,,inapparente Infektion“. Und weiterhin findet eine längere Virusausscheidung, wie sie in der Beobachtung von LÉpINE vorlag, durchaus ihren Platz im Rahmen eines noch nicht abgeschlossenen mehrphasigen Infektionsgeschehens, ohne daß dieses als chronisch bezeichnet werden kann. Dies ist durch die beispielhaften Untersuchungsergebnisse von Horstmann, Ward u. Melnick [315] bewiesen; bei manifest erkrankten Poliomyelitis-Patienten wurden während der ersten beiden Krankheitswochen bei $70 \%$, während der 3. und 4. Woche bei $50 \%$, während der 5 . und 6 . Woche bei $27 \%$, während der 7 . und 8 . Woche bei $12,5 \%$ eine Virusausscheidung im Stuhl, nie aber ein bleibendes Virusträgertum beobachtet. Im Hinblick auf eine, wenn auch bisweilen säumige Autosterilisation sei auf eine Studie über Poliomyelitis-Virusträger von MCCluRE u. LANGMuIR [467] verwiesen; bei 4 von 5 Abortivfällen und bei 20 von 27 Kontaktpersonen wurde das Virus aus dem Stuhl isoliert, dagegen nicht bei 4 weiteren Kontaktpersonen, deren Anamnese durch eine geheilte ,Poliomyelitisattacke“ belastet war. Der Beweis eines echten ,gesunden Dauerausscheider"-Zustandes ist noch nicht gesichert; wenn GEBHARDT u. Wordes [230] bei einem gesunden Manne auf Grund zweier im Abstand von 11 Monaten durchgeführter Virusnachweisversuche aus dem Stuhl mit positivem Ergebnis auf ein Ausscheidertum schließen, so muß ein solcher Befund dem kritischen Einwand gegenübergestellt werden, daß bei dieser Beobachtung vor allem anderen der Ausschluß mehrfacher, voneinander unabhängiger Infektionen versucht werden müßte.

Grundsätzlich ist die Möglichkeit einer gelegentlich verzögerten Autosterilisation des Wirtes und eines längeren Virusausscheiderzustandes nicht abzulehnen. Auf Grund von verlängerten Virusnachweisen bei wenigen gesunden Personen ist die Frage eines Trägertums auch von Kramer [40\%] erörtert und von Ward u. Sabin [729] als Ursache einer 
Verschleppung der Viren von einer Saison in die nächste diskutiert worden. Derartige Vorstellungen werden gestützt durch experimentelle Untersuchungen von Ackerman u. Kurtz [4]; diese Autoren haben festgestellt, daß in der Poliomyelitis-virusinfizierten HeLa-Zellkultur unter geeigneten Bedingungen die Zugabe von Immunserum einen cytopathogenen Viruseffekt zwar inhibiert, das Virus in den Zellen jedoch nicht inaktiviert zu werden braucht. Im ganzen gesehen bewegen wir uns aber in der Frage des stummen und andauernden ,Trägertums" bei der Poliomyelitis-Virusinfektion schon deshalb auf einem unsicheren Boden, weil der Einwand gegen die Fragwürdigkeit eines reinen Trägerzustandes bei Infektionen mit obligaten Zellschmarotzern überhaupt schwerlich zu entkräften sein dürfte. Aber auch bei Bejahung möglicher Trägerzustände als ,,latenter Infekt in seiner epidemiologischen Auswirkung“ (DoERR) ist nicht die Alternative ,Trägertum oder inapparente Infektion“" Gegenstand einer Diskussion, sondern bestenfalls ,,Trägertum in der Folge einer inapparenten oder apparenten Infektion". Damit wird die Anerkennung der virologisch, immunologisch, klinisch und teils auch morphologisch fundierten echten inapparenten Infektion aber nicht angetastet.

Die am Beispiel der Poliomyelitis vorgetragenen Überlegungen gelten sinngemäß auch für die anderen von uns bereits aufgeführten VirusWirtsbeziehungen, welche in Form einer akuten cyclischen Erkrankung klinisch manifest werden können. Diametral entgegengesetzt zu der Situation bei Infektionen mit Erregern höherer biologischer Ordnungsklassen ist das Trägertum für die von uns angeführten Agentien vorerst noch eine von Fall zu Fall zu beweisende Besonderheit, während die echte inapparente Infektion die Regel unterschwelliger Wirts-ErregerAuseinandersetzungen darstellt.

Bisher wurde der Virusträger-Zustand mit einer Besiedelung des Rachenraumes oder des Verdauungskanales, bzw. mit einer Virusausscheidung gleichgesetzt; darin erschöpft sich aber der Begriff des Trägertums in der anfangs zitierten weitesten Fassung DoerRs nicht. Es bleibt z. B. noch die Möglichkeit in Betracht zu ziehen, daß in einem Trägerzustand eine stumme und anhaltende Virus-Wirtsbeziehung in empfänglichen, nicht dem ,,milieu externe“ zugewandten Parenchymen vorliegen könnte. Ist ein solcher Zustand zeitlich begrenzt, so gilt grundsätzlich das gleiche, was wir bereits für die Situation des ausscheidenden Trägers und deren Limitierung durch die progrediente Autosterilisation gesagt haben. Anders lägen die Dinge bei einer praktisch unbegrenzten Besiedelungsdauer mit aktivem Virus. So verweist z. B. Bieling [\%0] zur Erklärung, daß das Tollwutvirus noch 25 Jahre nach einer (durch $\mathrm{Bi}$ erfolgten) Inoculation die manifeste Erkrankung auslösen könne, darauf, daß ,,langlebiges Poliomyelitis- und Lyssavirus“ an den ,,besonders langlebigen Ganglienzellen“ fixiert sein könne. „Man 
wird vermuten können,“ so folgert BIELING, ,daß eine solche latente Infektion nach stummer oder auch manifester Durchseuchung wesentlich dafür sein kann, daß ein Krankheitsvirus, das nur auf den Menschen angepaßt ist, sich in der seuchenfreien Zeit hält" . Die Beweisführung des angegebenen Beispieles der latenten Lyssa-Infektion verlangt unseres Erachtens allerdings 2 Klarstellungen: Einmal den sicheren Ausschluß einer eventuell ganz unauffälligen Neuinfektion in Zeiteinheit mit der ausgebrochenen Erkrankung und außerdem den Nachweis der LyssaVirusinoculation bei der Bißverletzung vor 25 Jahren. Da aber nun derartige Raritäten einer kritischen Betrachtung häufig nicht standhalten, erscheint es uns ratsam, auf ihnen nur dann eine Vorstellung zu entwickeln, wenn sie durch andere, lückenlose Beweise gestützt werden. Dies ist aber vorerst bei der Poliomyelitis nicht der Fall. Bei der Rabies wird die Frage eines echten, nicht infektiösen Trägertums beim Menschen unentschieden bleiben müssen, solange noch nicht einmal feststeht, welche Beziehungen die als Beleg für derartige Zustände angeführten Negri-Körperchen zu dem Virus haben. Eine Diskussion über die Unterscheidung der inapparenten Infektion von einem echten parenchymatösen Trägerzustand ist außerdem bei unseren heutigen Kenntnissen praktisch gegenstandlos; nach allem bisher Gesagten läßt nämlich eine WirtsErregerbeziehung, welche der Möglichkeit einer inapparenten Infektion in der vorgetragenen Definition Raum gibt, schwerlich die Realisierung eines echten andauernden Trägertums zu.

Nun gibt es aber noch andere Formen stummen viralen Infektionsgeschehens, deren symptomatische Charakteristik Parallelen zur inapparenten Infektion aufzuweisen scheinen. Wir führen als Prototyp dafür die Theiler-Virusinfektion der Maus [682] an. Die meisten Mäusezuchten sind weitgehend, bzw. 100\% ig mit diesem Agens durchseucht [511, 512; 75\%]. Das von den Jungmäusen aufgenommene und im Darm beherbergte Virus wird über 2-6 Monate ausgeschieden; danach sind die Tiere im allgemeinen virusfrei. Die intestinale Infektion scheint keine obligate Virämie zur Folge zu haben. Tritt diese ein und wird der Erreger auch in das ZNS eingebracht, so bildet sich eine Encephalomyelitis aus; nach OLITzKy [511] kommt dieses, durch intracerebrale Inoculation auch bei enteral durchseuchten Mäusen reproduzierbare Ereignis in einem Verhältnis zur Darminfektion von 1:1000 bis 1:4000 vor. Bei Theilervirusfreien Zuchten erwies sich der Kontagionsindex als ganz erheblich höher [188; 450], teils sogar betrug er 100\% [452]. Das Wesen dieser Infektion scheint nun, soweit das heute bekannt ist, darin zu beruhen, daß die isolierte stumme Darmbesiedelung [683] der mit einer Virämie verbundenen akuten oder auch chronischen [14\%] zentralnervösen Erkrankung gegenübersteht. Der Nachweis von neutralisierenden Antikörpern im 
Serum enteral infizierter Mäuse $[683 ; 150]$ sowie die erheblich größere Virusanfälligkeit von Mäusen aus Theilervirus-freien Mäusezuchten einerseits, und der erwiesene latente Virusbefall des ZNS torpid erkrankter Tiere (Theiler [682]; Daniels [14\%] u. Mitarb.) andererseits ist im Verein mit der experimentell durch intracerebrale Virusinoculation möglichen Auslösung der akuten Encephalomyelitis bei enteral bereits latent durchseuchten Tieren Grundlage der folgenden Beurteilung: Es liegt bei der Maus eine große Empfänglichkeit für das Theiler-Virus vor; durch eine Antikörper-Mitgift von einer Generation zur anderen wird in durchseuchten Zuchten die natürliche Infektion der Tiere auf einen blanden und asymptomatischen Reaktionskontakt in enteralen Bezirken herabgemindert, welcher allerdings eine zusätzliche Antikörperbildung zur Folge hat. Dies ist aber nicht gleichbedeutend mit der Erlangung einer Immunität. Denn wenn die von den Antikörpern gestützte Barriere der enteralen Bereiche durchbrochen wird und - im natürlichen Infektionsgang — die virämische Erregeraussaat stattfindet, dann tritt die manifeste Erkrankung der Encephalitis ein. Mit einer inapparenten Infektion hat dieses ganze pathogenetische Prinzip nichts zu tun. Denn wenn auch die manifesten Erkrankungsformen unberücksichtigt bleiben können, so liegt im Falle der stummen Darmbesiedelung weder eine ,maladie aigue septicémique“ vor, noch hinterläßt dieser Prozeß eine ,immunité plus ou moins durable“.

Eine in grundsätzlichen Zügen der Theiler-Virusinfektion der Maus vergleichbare Situation finden wir auch bei der ubiquitären [120; u. a.] Virusdurchseuchung des Menschen mit dem Herpes-Virus. Auch hier steht ein - möglicherweise auf die Außenbezirke einschließlich der ,,inneren Außenfläche“ des Organismus beschränkter stummer WirtsErregerkontakt der manifesten herpetischen Läsion gegenüber; wir können in diesem Zusammenhang die Zweifel an der kontagiösen Ausbreitung und Haftung des Herpes-Virus, wie sie von DoERr [162] geäußert wird, unberücksichtigt lassen, da hierdurch derGegenstand unserer Betrachtung nicht wesentlich berührt wird. Sicher steht nämlich, daß das Kollektiv der Wirtsorganismen mit zunehmendem Lebensalter in steigendem Maße Antikörper gegen dieses Agens bildet $[9 \% ; 114 ; 148$; $22 \%$; 234; u. a.], ohne eine wirkliche Immunität zu erwerben. Nach Untersuchungen von ZuRukzoglu u. Hruszex [\%59] scheint das Virus an Bereiche abgeheilter Herpes-Eruptionen sogar besonders leicht fixiert zu bleiben; damit würde selbst die Annahme einer lokalen Immunität hinfällig. Entsprechend der Situation bei der Theiler-Virusinfektion und auch der Herpes-Virusinfektion der Maus [3\%1] scheint die Anfälligkeit des Menschen gegenüber dem Herpesvirus im jüngsten Lebensalter am größten zu sein, wie man dies aus vereinzelten Berichten schwerster und tödlicher herpetischer Allgemeininfektionen neugeborener Kinder entnehmen kann $[203 ; 426 ; 469 ; 558 ; 758]$. Ohne daß wir auf die sich hier stellenden 
Probleme eingehen müssen, können wir schon aus diesen wenigen skizzenhaften Zügen der Beziehungen zwischen dem Menschen und dem Herpesvirus deren Bedeutungslosigkeit für die inapparente Infektion ersehen.

Den Phänomenen der herpetischen Infektion könnten wiederum die Gegebenheiten der Cytomegalie gegenübergestellt werden. Auf Grund des histomorphologischen Kriteriums eines Einschluß-Nachweises in den Zellen der meisten Parenchyme wird dieser Status als Virusinfektion angesehen. Nach bisher vorliegenden Berichten ist dieser auch beim Tier (Affennachweise durch Vogel u. Pinkerton [721]) erhobene Befund beim Menschen, vorzüglich bei atrophischen, aus vielerlei Ursachen gestorbenen Säuglingen zu erheben [60\%]. Daneben liegen allerdings auch Beschreibungen frühkindlicher Todesfälle vor, in welchen eine generalisierte Cytomegalie als tödliche Krankheit dargestellt, oder als Ursache schwerer Mißbildungen angesehen wird $[6 ; 51 ; 136 ; 161 ; 2 \% 6 ; 368 ; \% 18]$. Es ist jedoch müßig, bei diesem vorerst noch sehr wenig erforschten Leiden die bisweilen schon anklingende Diskussion über eine eventuelle unterschwellige Infektion auf die ,,inapparente Infektion“ auszudehnen.

Ein noch wenig erschlossenes Gebiet der stummen Virus-Wirtsbeziehungen liegt in den Infektionen mit den ,,maskierten Viren“" und den sogenannten ,,virus endormis" vor uns. Wir haben im 1. Kapitel schon einige näher erforschte Infektionen dieser Art genannt. Bei den meisten derartigen Wirts-Viruskontakten, zu denen außer den bereits aufgezählten Vertretern in erster Linie Tumor-erzeugende Agentien (siehe bei GönNeRT [240]) gehören, wird durch unterschiedliche unspezifische Provokationen des Wirtes die beschädigende Wirkung des Erregers manifest [217]. Auch hier sind keine Beziehungen mit einer inapparenten Infektion unserer Begriffsbildung aufzuzeigen.

Dagegen begegnet uns in der Pathogenese der Schweineinfluenza ein im Rahmen dieser Besprechung sehr interessantes Problem. Die außerordentlich verdienstvollen Studien SHopes [614-619] haben aufgezeigt, daß das ätiologische Agens dieser Zoonose ein Virus ist, welches als latenter Parasit in Schweinelungenwürmern existiert. Wenn nun Schweine derart infizierte Lungenwürmer (metastrongylus elongatus) beherbergen, so können sie - provoziert durch das Bacterium H. influenzae suis - trotz ganzjährigem Kontakt mit dem Virus ausschließlich in Herbst- und Wintermonaten an der Schweine-Influenza erkranken. Soweit wäre dieser vielschichtige Komplex eine Gegebenheit, die mit einer inapparenten Infektion nichts zu tun hätte. Neueste Untersuchungsergebnisse von SHOPE [619] haben nun aber gezeigt, daß bei einer experimentellen Reproduktion der Schweineinfluenza, unter Berücksichtigung aller genannten Faktoren, nur ein Teil der Tiere manifest erkrankt, während andere eine nur serologisch erfaßbare unterschwellige 
Reaktion durchmachen; grundsätzlich wäre also hier die kuriose Situation in Betracht zu ziehen, daß eine ,maskierte“ Virusinfektion bei ihrer ,Demaskierung" fakultativ in Form einer inapparenten Infektion verläuft.

Dies ist aber keineswegs die einzige Form einer solchen Querverbindung im System der Phänomenologie viraler Infektionen. Es zeichnen sich solche Linien auch bei der Ektromelie der Maus ab, deren gänzlich unterschiedliche Infektionsverläufe von dem kontinuierlich stummen und reaktionslosen Parasitismus bis zu akut ablaufenden cyclischen Erkrankungsformen reichen [190-192]. Auch die epizootische Choriomeningitis-Virusinfektion der Maus existiert meist in Form einer erscheinungsfreien latenten Besiedelung; sie kann aber stets durch unspezifische Provokationen, wie Injektionen von Fremdserum [424], Stärkelösung [194] oder steriler Bouillon [696] aktiviert und zur manifesten Erkrankung gesteigert werden. Neben dieser, angeblich einer intrauterinen Übertragung folgenden latent-parasitären Beziehung gibt es laut der bereits zitierten Mitteilung von Wiтhney [\%40] und kürzlich publizierter Untersuchungen von HAAS [253] bei späteren Virusübertragungen eine Vielfalt von anderen akuten und chronischen, manifesten und unterschwelligen Infektionsverläufen; dies ist im Hinblick auf die humanen Infektionen mit Erregern aus der Gruppe des Choriomeningitisvirus insofern beachtenswert, als die Erkrankung des Menschen einen akut cyclischen Charakter hat und weil auf Grund von immunologischen Studien im Verlaufe einer lokalen Epidemie [\%3], sowie der kasuistischen Mitteilung einer vermutlich diaplacentaren Infektion [389] ohne typische Erkrankung der Schwangeren Hinweise für das Vorkommen echter inapparenter Infektionen beschrieben sind.

So interessant und reizvoll die Verfolgung dieser feinen Abstufungen in den pathogenetischen Ursachen derartiger Infektionen ist - sie sprengt die Grenzen einer ordnenden Betrachtung, die wir in diesem Kapitel bezwecken. Wir werden aber in anderem Zusammenhang noch einmal darauf stoßen.

Mit den bisher behandelten Formen stummer Virus-Wirtsbeziehungen sind aber die Möglichkeiten symptomloser Prozesse, welche von der inapparenten Infektion abgegrenzt werden müssen, nicht erschöpft. Auch bei einer Anzahl von unterschwellig verlaufenden, infektionsbedingten Reaktionen prämorbiden Charakters ist die Klarstellung der Unterschiede zu der inapparenten Infektion angezeigt. Derartige Geschehen können im wesentlichen in 2 Gruppen zusammengefaßt werden; für jede soll ein modellhaftes Beispiel angeführt werden.

So diskutiert BREIG [102] für die Poliomyelitis die Möglichkeit „,prämorbider, latenter und chronisch weiterschwelender Infektion des ZNS“. Einer solchen Vorstellung muß unter Vorgriff auf die getrennte 
Besprechung der Folgen einer inapparenten Infektion entgegengehalten werden, daß $\mathrm{J}_{\mathrm{ACOB}}[330,331]$ nachdrücklich auf die den ,,postpoliomyelitischen Zustandbildern" zu unterstellenden, Jahre nach dem Infektionsablauf klinisch manifest werdenden postpoliomyelitischen Angiopathien aufmerksam gemacht hat; die Annahme einer direkten Beteiligung des Poliomyelitisvirus an derartigen Prozessen widerspricht aber allen gewonnenen Vorstellungen von dem Wesen der Auseinandersetzung des Menschen mit diesem Agens. Damit sind auch derartige reaktive Geschehen eindeutig von der inapparenten Infektion zu trennen; grundsätzlich könnten sie allerdings von Fall zu Fall apparente Spätfolgen einer vorausgegangenen inapparenten Infektion sein. Die Diskussion derartiger Fragen gehört aber - wie schon gesagt — in das Kapitel der Auswirkungen einer inapparenten Infektion.

Hinweise für eine unterhalb der Grenzlinie klinischer Symptomatik ablaufende Virusinfektion treten dem Arzt auch immer wieder bei der Virus-Hepatitis entgegen. Zahlreiche Untersuchungen bezeugen, daß in der Umgebung von Hepatitiskranken bei ,,gesunden“ Personen Stoffwechselstörungen nachzuweisen sind, welche das Vorliegen eines, wenn zwar unterschwelligen so doch für die Virushepatitis typischen Leberparenchymschadens vermuten lassen [2\%\%; 383; 669; 68\%; 747]. Vergleiche solcher Untersuchungsergebnisse mit bioptischen Gewebskontrollen sowie virologischen Studien [498; 671] und experimentellen Reproduktionen derartiger Infektionen an Versuchspersonen bestätigen diese Annahme [495; u. a. m.]. Sie bejahen außerdem die Möglichkeit eines langdauernden Aufenthaltes des aktiven Virus in solchen Individuen, - eine Situation, welche ja durch die Möglichkeit yatrogener Infektionen große Bedeutung erlangt hat: es wurden dabei Virusausscheidungen in Zeitspannen bis zu $51 / 2$ Jahren verfolgt. STokes u. Mitarb. vermuten, daß solche als ,Trägertum“ bezeichneten Zustände durch eine Unfähigkeit des Wirtes, eine wirksame Immunität in früheren Stadien der Infektion zu schaffen, bedingt sind. Im allgemeinen ist aber eine zur Immunität ausreichende Antikörperbildung zumindestens bei der Virushepatitis-A (Hepatitis infectiosa) möglich; dies läßt der therapeutische und prophylaktische Effekt von Gamma-Globulin in entsprechenden Situationen erkennen [172; 231; 319]. Für die Virushepatitis-B (Serumhepatitis) wird ein Schutz durch Gamma-Globulin von Stokes [6\%0] bestritten. Der Versuch einer Profilierung der pathogenetischen Prinzipien aller dieser Phänomene läßt vorderhand nur die Annahme folgender Möglichkeiten zu: Ein Teil der Hepatitis-Virusinfektionen des Menschen verläuft in Form einer akut-cyclischen Erkrankung mit nachfolgender protektiver Immunität; andere Prozesse dieser Genese schwelen mangels ausreichend wirksamer Immunisierung chronisch weiter; daneben ist eine durch schädigende Auswir- 
kungen nicht belastete stumme Virusbesiedelung empfänglicher Wirtszellen in Betracht zu ziehen; und schließlich ist von Fall zu Fall an die Möglichkeit einer von dem infektiösen Prozeß losgelösten Progredienz des Leberschadens als Folge einer Virusläsion des Leberparenchyms zu denken. Die Hepatitis-Virusinfektion würde damit alle uns interessierenden Möglichkeiten einer Wirts-Virusbeziehung in sich vereinen. Die Realisierung einer echten ,,inapparenten Infektion“ ist aber nur für die erstgenannte Form eines Reaktionsablaufes in Erwägung zu ziehen. So wenig sie dafür von vornherein abzulehnen ist, so sehr fehlen uns aber bisher ausreichende Beweisunterlagen für ihr Vorkommen.

Rekapitulieren wir nun abschließend unseren Überblick über die Stellung der ,inapparenten Infektion“ innerhalb der angeführten Virusinfektionen, so kommen wir zu folgender

\section{Zusammenfassung}

Eine ,inapparente Virusinfektion“ ist unter Zugrundelegung unserer heutigen Kenntnisse über die Pathogenese der Viruskrankheiten ausschließlich für jene Infektionen vorbehalten, bei welchen die WirtsErreger-Auseinandersetzung in Form eines akuten cyclischen Prozesses erfolgt, und wobei eine echte Immunisierung gegen das infektiöse Agens eintritt. Hierfür können zahlreiche experimentelle, klinische und immunologische Beispiele vorgelegt werden. Die zusätzliche Annahme eines echten ,Trägertums“ ist bei derartigen Infektionen eine contradictio in adjectu. Stumme Virusinfektionen, welchen keine echte Immunität folgt und wie sie z. B. bei der Theiler-Virusinfektion der Maus, bei der menschlichen Herpes-Virusinfektion oder bestimmten Formen der Virus-Hepatitis vorliegen können, erfüllen das Postulat der ,,inapparenten Infektion" nicht. Progrediente, vom eigentlichen infektiösen Vorgang losgelöste und zunächst unterschwellig sich entwickelnde Prozesse im Gefolge einer Virusinfektion, wie sie bei den post-poliomyelitischen Zustandbildern oder bei Spätfolgen einer Virushepatitis in Erscheinung treten können, sind ebenfalls von dem Begriff der ,,inapparenten Infektion" zu trennen.

\section{Ursachen der Apparenz oder der Inapparenz einer Virusinfektion}

\section{Die Ausgangsbasis einer Unterscheidung der Ursachen von Apparenz und Inapparenz einer Infektion}

Die Frage nach der pathogenen Wirksamkeit eines infektiösen Agens bzw. nach den Gründen des Fehlens nosologischer Auswirkungen einer Infektion geht von der Klinik aus und stellt das Verhalten des infizierten 